\title{
Distribution and evolution of glycoside hydrolase family 45 cellulases in nematodes and fungi
}

Juan E Palomares-Rius ${ }^{1,2+}$, Yuuri Hirooka ${ }^{3,4+}$, Isheng J Tsai ${ }^{1}$, Hayato Masuya ${ }^{3}$, Akina Hino ${ }^{1}$, Natsumi Kanzaki ${ }^{3}$, John T Jones ${ }^{5,6}$ and Taisei Kikuchi, ${ }^{1,3 *}$

\begin{abstract}
Background: Horizontal gene transfer (HGT) has been suggested as the mechanism by which various plant parasitic nematode species have obtained genes important in parasitism. In particular, cellulase genes have been acquired by plant parasitic nematodes that allow them to digest plant cell walls. Unlike the typical glycoside hydrolase (GH) family 5 cellulase genes which are found in several nematode species from the order Tylenchida, members of the $\mathrm{GH} 45$ cellulase have only been identified in a cluster including the families Parasitaphelenchidae (with the pinewood nematode Bursaphelenchus xylophilus) and Aphelenchoididae, and their origins remain unknown.
\end{abstract}

Results: In order to investigate the distribution and evolution of GH45 cellulase genes in nematodes and fungi we performed a wide ranging screen for novel putative GH45 sequences. This revealed that the sequences are widespread mainly in Ascomycetous fungi and have so far been found in a single major nematode lineage. Close relationships between the sequences from nematodes and fungi were found through our phylogenetic analyses. An intron position is shared by sequences from Bursaphelenchus nematodes and several Ascomycetous fungal species.

Conclusions: The close phylogenetic relationships and conserved gene structure between the sequences from nematodes and fungi strongly supports the hypothesis that nematode GH45 cellulase genes were acquired via HGT from fungi. The rapid duplication and turnover of these genes within Bursaphelenchus genomes demonstrate that useful sequences acquired via HGT can become established in the genomes of recipient organisms and may open novel niches for these organisms to exploit.

Keywords: Bursaphelenchus, Cellulases, Horizontal gene transfer, Ascomycota, Fungi

\section{Background}

Cellulose, a polymer of $\beta$-1,4-linked glucose molecules, is the major polysaccharide component of plant cell walls and is the most abundant organic polymer on Earth. Many microorganisms produce cellulases to degrade cellulose in order to use it as a carbon source. For plant pathogens, the plant cell wall is the primary barrier that they need to overcome and the production of enzymes capable of

\footnotetext{
* Correspondence: taisei_kikuchi@med.miyazaki-u.ac.jp

${ }^{\dagger}$ Equal contributors

'Division of Parasitology, Faculty of Medicine, University of Miyazaki, Miyazaki 889-1692, Japan

${ }^{3}$ Forestry and Forest Products Research Institute, Tsukuba, Ibaraki 305-8687, Japan

Full list of author information is available at the end of the article
}

degrading cellulose is therefore of critical importance for colonization of plants.

Most animals (Metazoa) do not have endogenous cellulases and rely instead on intestinal symbiotic microorganisms for cellulose digestion. However, recent studies have shown that some insects and plant-parasitic nematodes have endogenous cellulases that degrade cellulose polymers $[1,2]$.

Cellulases can be grouped into families based on their sequence and on the basis of hydrophobic cluster analysis [3]. Fourteen families of glycosyl hydrolases (GH) are known to include proteins that degrade cellulose (http://www.cazy.org). It is thought that proteins within each group are structurally related and are likely to have evolved from a common ancestor [4]. 
Cellulases from two distinct glycosyl hydrolase families (GH5 and GH45) have been found in nematodes. GH5 cellulases have been found in a wide range of Clade 12 Tylenchid plant parasitic nematodes and show relatively high similarity to bacterial GH5 sequences, leading to the suggestion that they were acquired via horizontal gene transfer (HGT) from bacteria [5-7]. However, another plant-parasitic nematode Bursaphelenchus xylophilus, which is located in Clade 10 as described by van Megen et al. [8] and is not directly related to the Clade 12 Tylenchid plant parasites, has GH45 cellulases rather than GH5 [9]. These two GH families show little amino acid similarity to each other and have distinct kinetic mechanisms, catalytic residues and three dimensional structures although both catalyze the breakdown of similar substrate; cellulose and hemicelluloses [10,11].

The origin of the nematode GH45 cellulases remains unclear, although HGT from fungi seems likely given the high similarity to fungal GH45 cellulases and the absence of sequences resembling GH45 cellulases from all other nematodes analysed to date.

Bursaphelenchus xylophilus is the causal agent of pine wilt disease [12]. In their pathogenic life cycle the nematode is transmitted from trees killed by pine-wilt to healthy pines by vector beetles. Once the nematodes enter the tree, they feed on plant cells in the tree, leading disruption of pine tissues and lethal wilt. As the pine wilts and dies, the nematodes start to feed on fungi that invade the dying tree. Furthermore, most Bursaphelenchus species are solely fungal feeders and all species rely on fungi as a food source at some stage of their life cycle.

In this study we have conducted a wide ranging screen and intensive phylogenetic analysis of GH45-like sequences in nematodes and fungi, particularly those found in association with plants. Our results show a wide distribution of GH45 cellulases in Ascomycetous fungi and a narrow but concentrated distribution in nematodes, a single lineage that includes a number of facultative plant parasites. The close relationships between the nematode and fungal sequences, as well as a shared intron position in some of the nematode and fungal sequences, suggest that nematode GH45 cellulases were acquired via HGT from fungi and subsequently underwent repeated duplication within nematode genomes.

\section{Results}

\section{Amplification of GH45 cellulase sequences}

Genomic DNA was extracted from 289 fungal species/ strains and 26 nematode species/strains (Additional file 1: Table S1, S2) and used for PCR amplification with a degenerate primer pair designed from a conserved region of known GH45 cellulases (Additional file 2: Figure S1). Clear bands were observed at around 500-2000 bp following agarose gel electrophoresis. The majority of successful amplifications were from Ascomycetous fungi or Bursaphelenchus nematode species. No amplification was seen with Basidiomycetous fungi or distantly related (Clade 12) nematode species including Aphelenchus avenae and Pratylenchus sp., suggesting the absence of GH45 in these species.

Sequence analysis revealed most of those fragments contained the conserved sequence of GH45 cellulases including two catalytic core residues (Asp, Asp) (Additional file 2: Figure $\mathrm{S} 1$ ). In total we obtained 47 sequences from 13 nematode species (out of 20 species tested) including B. doui, B. conicaudatus, B. purvispicularis, B. xylophilus, B. mucronatus, B. luxuriosiae, B. okinawaensis, B. kiyoharai, Bursaphelenchus sp1, Bursaphelenchus sp2, B. yongensis and B. poligraphi (Additional file 1: Table S3). Ruehmaphelenchus sp. in this study and Aphelenchoides besseyi [13] in a previous study are the only species other than Bursaphelenchus species from which GH45-like sequences were identified. For fungi 70 GH45-like sequences were identified from 61 fungal species (out of 259 species/strains tested), all of which belong to Ascomycota (Additional file 1: Table S4).

\section{Gene structures}

The nematode GH45 sequences can be broadly grouped into two different intron-types, some of which were successfully confirmed by RT-PCR or RNA-seq data (Additional file 1: Table S3): genes with no intron (p0) and genes with one intron at position 11 (P11) (Figure 1, Additional file 1: Table S3). Position 11 introns in the nematodes varied in length from $36 \mathrm{bp}$ to $220 \mathrm{bp}$ (Additional file 1: Table S3). Some nematode species contained more than one GH45 sequence and included both P0 and P11 intron-types. These species included B. xylophilus, B. doui, $B$. purviscularis, $B$. mucronatus and $B$. luxuriosae. We were only able to amplify one intron-type from other species: $B$. conicaudatus, B. kiyoharai and Bursaphelenchus sp1 contained only GH45 sequences with P11 introns while Bursaphelenchus sp3, B. okinawaensis, B. yongensis, B. poligraphi and Bursaphelenchus sp2 contained only p0 GH45 sequences. Only intronless sequences were identified from Ruehmaphelenchus sp.

Eleven GH45 genes were predicted in the recently published B. xylophilus genome sequence [14]. Six of these 11 genes have no intron (p0), 2 genes have introns at position 11 (p11) and 3 genes at position 8 (p8) (Figure 1, Additional file 1: Table S3).

Eleven intron positions were found in the fungal sequences. Most of the fragments contained only one intron (Figure 1). Forty-three sequences had one intron at position 6,15 sequences had one intron at position 11 and 10 had no intron in the amplified fragment. The intron lengths were $46 \mathrm{bp}$ to $343 \mathrm{bp}$. One intron position (p11) was shared by the nematodes and several fungal species. 


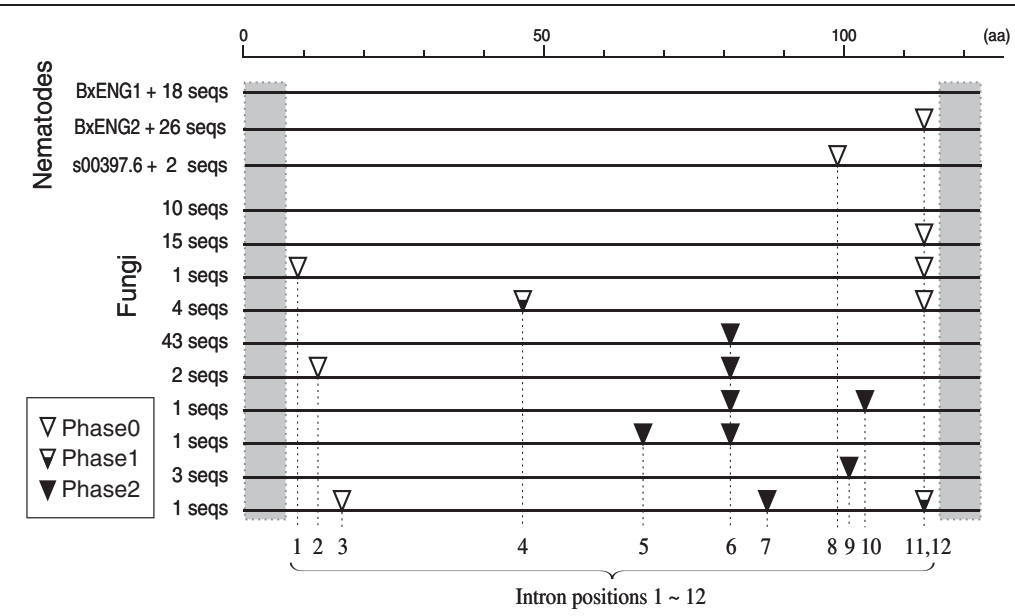

Figure 1 Intron/exon structures of GH45 fragments of nematodes and fungi. Intron positions found in nematode and fungal sequences are shown by triangles on a simplified amino acid alignment of GH45 proteins (the original alignment for some specific species can be found in Additional file 2: Figure S1). Conserved regions used to design primers are box shaded. Phase of the introns is shown by distinct triangles. Thirteen intron positions were found in total from nematodes and fungi and position 11 is shared by fungi and nematode sequences.

\section{Phylogenetic analysis Nematode sequences}

To reconstruct the evolutionary relationship between the species with successful amplified GH45 sequences, a phylogenetic tree was built based on nearly full length $18 \mathrm{~S}$ ribosomal RNA (rRNA) gene from these species. A comprehensive list of nematodes including the superfamily Aphelenchoidea, the order Tylenchida and some members of Cephaloboidea were used for the analysis. However, a major effort in sequences for phylogenetic reconstruction was done for members of Aphelenchoidea, which harbour GH45 sequences (Figure 2).

The phylogeny of the Bursaphelenchus genus is characterised by a well defined clade (100 PP) which includes B. luxuriosae, B. xylophilus, B. mucronatus, B. doui, B. conicaudatus, and will be referred as the "xylophilus group" throughout the manuscript. B. kiyoharai and Bursaphelenchus sp1 are clustered into a clade next to that of the xylophilus group (100 PP). The rest of the species considered in the study are distributed throughout the different subclades formed.

Phylogenetic trees of nematode GH45 nucleotide sequences are shown in Figure 3 with two fungal GH45 sequences included as outgroup. Similar topology was shown using amino acid sequences (Additional file 2: Figure S2). The phylogeny revealed that the xylophilus group clustered into one large clade comprised by two well supported subclades: subclade 1 is comprised by sequences with intron 11 (p11) and subclade 2 with sequences that show other intron positions (p0 or p8). Sequences from B. okinawaensis are clustered together with those from B. kiyoharai and Bursaphelenchus sp1 which comprise a sister clade of the xylophilus group in the SSU tree. Bursaphelenchus sp3 sequences were clustered into an independent clade at the basal position. The sequences from Ruehmaphelenchus sp. were positioned inside the Bursaphelenchus sequences with a long branch; a similar pattern is also observed in the SSU tree.

Ten out of 11 B. xylophilus GH45 genes from the genome sequence of this species were clustered into four clades in the aforementioned large clade. These clades were composed of the following sequences: i) Bx-eng-2 and Bx-eng-3 with B. purvispicularis and B. mucronatus sequences; ii) s01038.221, Bx-eng-1, s01116.1 and s00119.44 with B. purvispicularis and B. mucronatus sequences; iii) s00397.15, s00397.16, s00397.6 and s01288.37 sequences and iv) s01147.110 sequence occupying a basal position with other Bursaphelenchus species sequences and $A$. besseyi. This close relationship between sequences for the same species is also observed for other species with the exceptions of B. yongensis and $B$. poligraphi from which we were able to identify only one and two GH45 sequences respectively.

The positions of the B. purvispicularis GH45 sequences differed greatly from the position of this species within the SSU phylogeny. The $A$. besseyi GH45 sequence [13] was nested within the sequences from the Bursaphelenchus species.

\section{Fungal sequences}

A phylogenetic tree based on the large subunit ribosomal RNA genes (LSU) of fungi is shown in Figure 4. A comprehensive set of species belonging to Ascomycota ranging from Sordariomycetes to Saccharomycetes were included in the phylogenetic tree. The topology of the tree correlated well with previously described phylogenetic data from fungi, even though we only sequenced a 


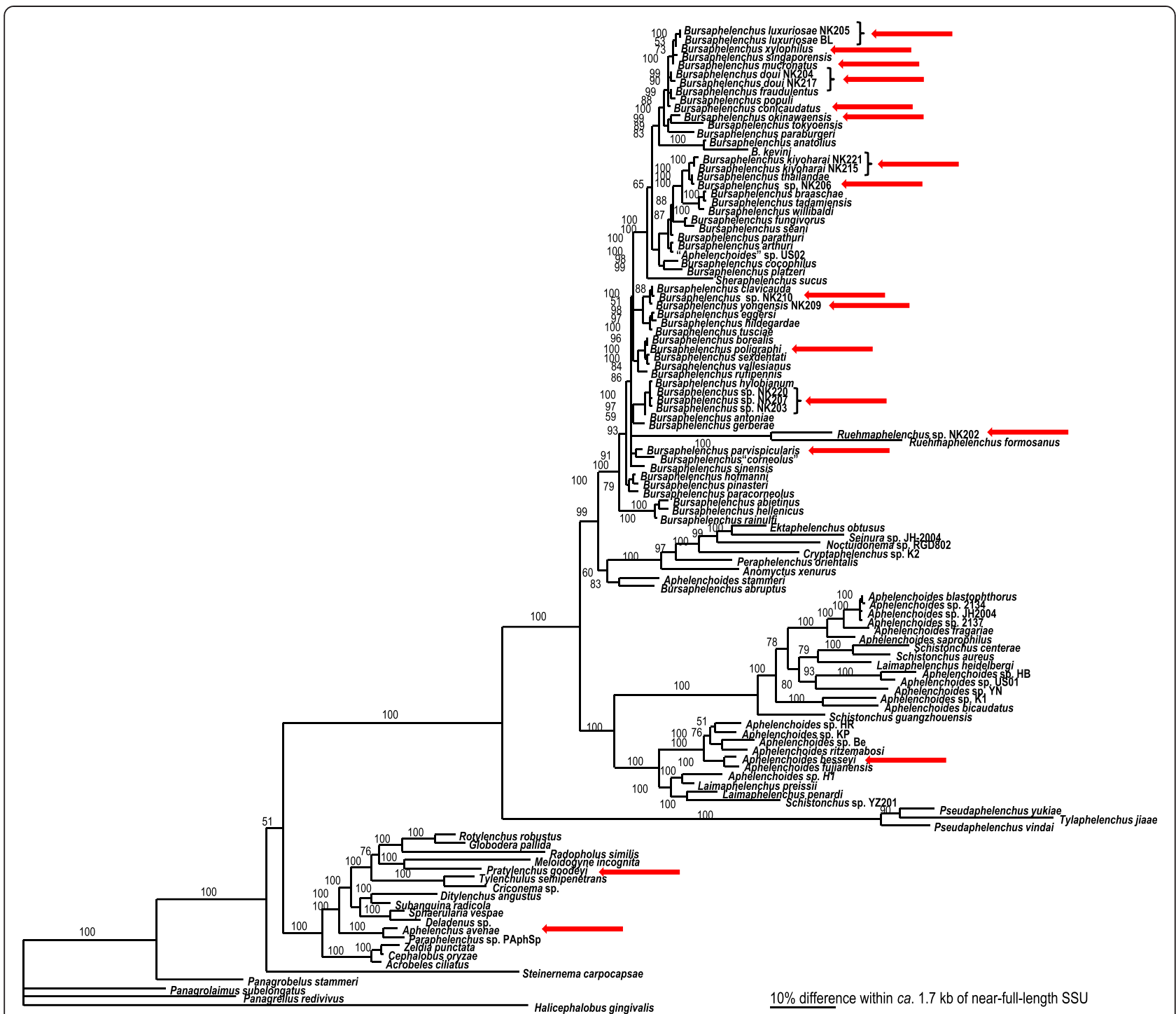

Figure 2 The molecular phylogenetic relationships among selected species of Aphelenchoidea, Tylenchoidea and Cephaloboidea. 10001st Bayesian tree inferred from near-full-length small subunit of ribosomal RNA gene under GTR + I $+G$ model. The figure has been adapted from the phylogenetic tree provided by Kanzaki \& Tanaka [15]. Arrows indicate species used in this study.

single locus and some groups were not represented by a large number of species e.g. [16-18]. The Sordariomycetes clade is well supported with $100 \mathrm{PP}$ and $97 \mathrm{BS}$ values. Leotiomycetes and Dothideomycetes were nearly monophyletic although Patellina sp. (02E05) did not nest in the main clade. Two species of Eurotiomycetes were grouped and made a distinct clade with low phylogenetic values. The other representative species of each class e.g., Pezizomycetes, Basidiomycetes and Agaricomycetes, were supported by high phylogenetic values and long branches. The fact that these species did not taxonomically correlate with previous phylogenetic data was most likely because we used only a single locus in this study.

A phylogenetic tree of GH45 sequences based on nucleotide sequences is shown in Figure 5 and the phylogenetic tree based on amino acid sequences is shown in Additional file 2: Figure S3. The trees of LSU and GH45 sequences were not completely consistent for some species, genera and classes, but showed a good consistency in general. In the phylogenetic trees of GH45 sequences, none of the classes we analysed were monophyletic, except for Agaricomycetes. Leotiomycetes was nearly monophyletic as Komagataella pastoris and Rhizina undulata were clustered with the Leotiomycetes clade in the phylogenetic trees of GH45 sequences. This class is also nearly clustered as monophyletic in the phylogenetic trees of amino acid sequences. Sordariomycetes were divided into two clades in the both phylogenetic trees. Interestingly, the two clades showed several genera or species duplications such as Pestalotiopsis glandicola, Bartalinia 


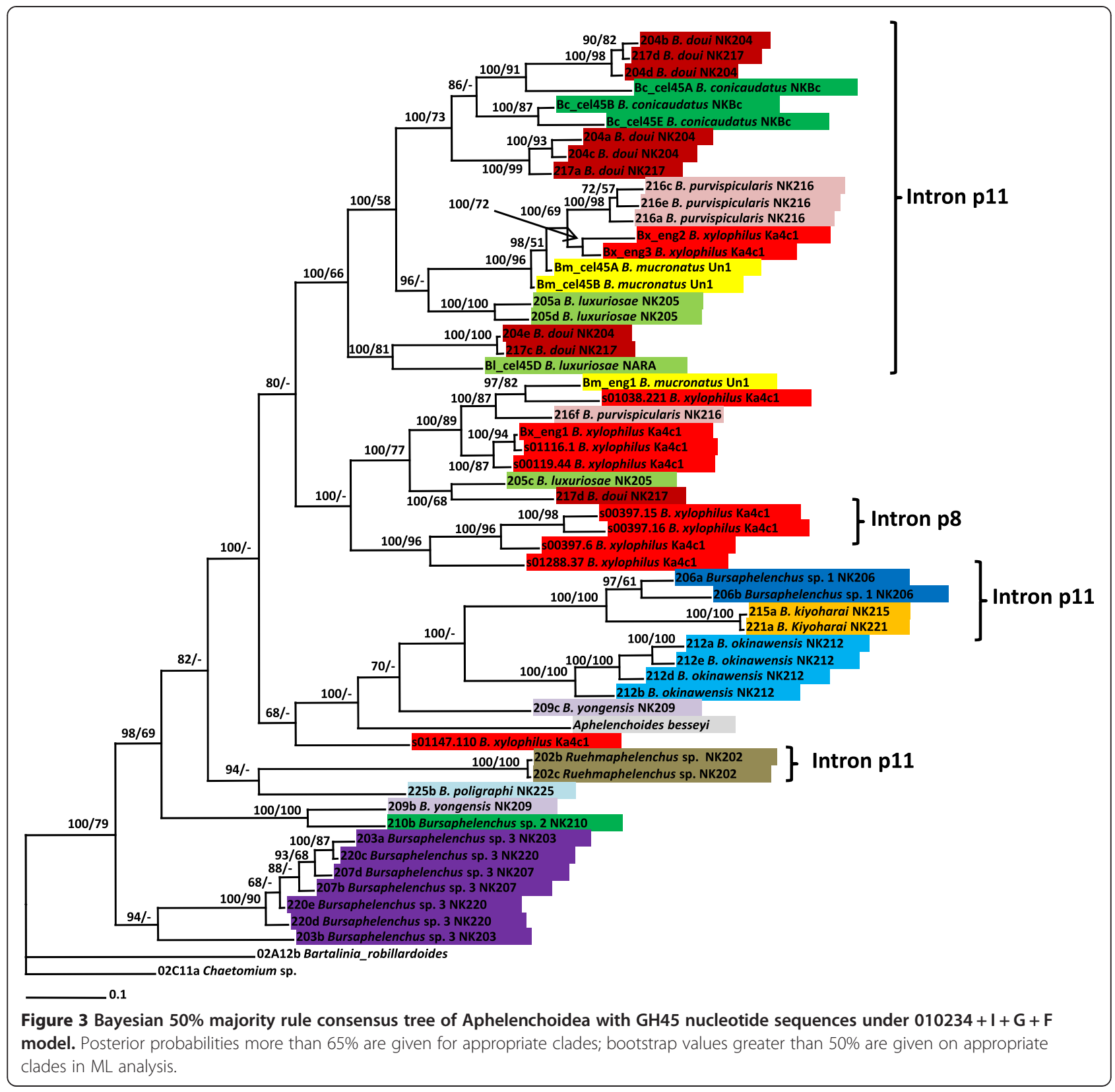

robillardoides, Monochaetia monochaeta and Humicola grisea. Dothideomycetes was divided into several clades that were not sorted by the general fungal taxonomy. Our Dothideomycetes data included four orders: Dothideales, Pleosporales, Capnodiales, and Botryosphaeriales, and none of the orders were monophyletic in the both phylogenetic trees. Although we used Mucoromycotina as a outgroup taxa in our trees, Ustilago maydis clustered with the Mucoromycotina clade.

\section{Combined tree}

A broad phylogenetic tree was generated using the GH45 sequences from nematodes and fungi obtained in this study and other sequences from CAZy database (http://www.cazy.org/). Sequences from bacteria and molluscs were not included as they are very different compared to sequences from other organisms (Figure 6). The ML best tree showed a monophyletic clade for nematodes and also for protists and insects, while the fungi sequences are distributed into several separated clades. However, the clades formed are weakly supported with the exception of prostists and insects. Deep clades are usually well supported in our analysis. Some sequences from fungi were located in the Nematoda clade (CAJ75963 [Rasamsonia emersonii]; CBX93072 [Leptosphaeria maculans]; AAF05700 [Alternaria alternate]). A fungal clade 


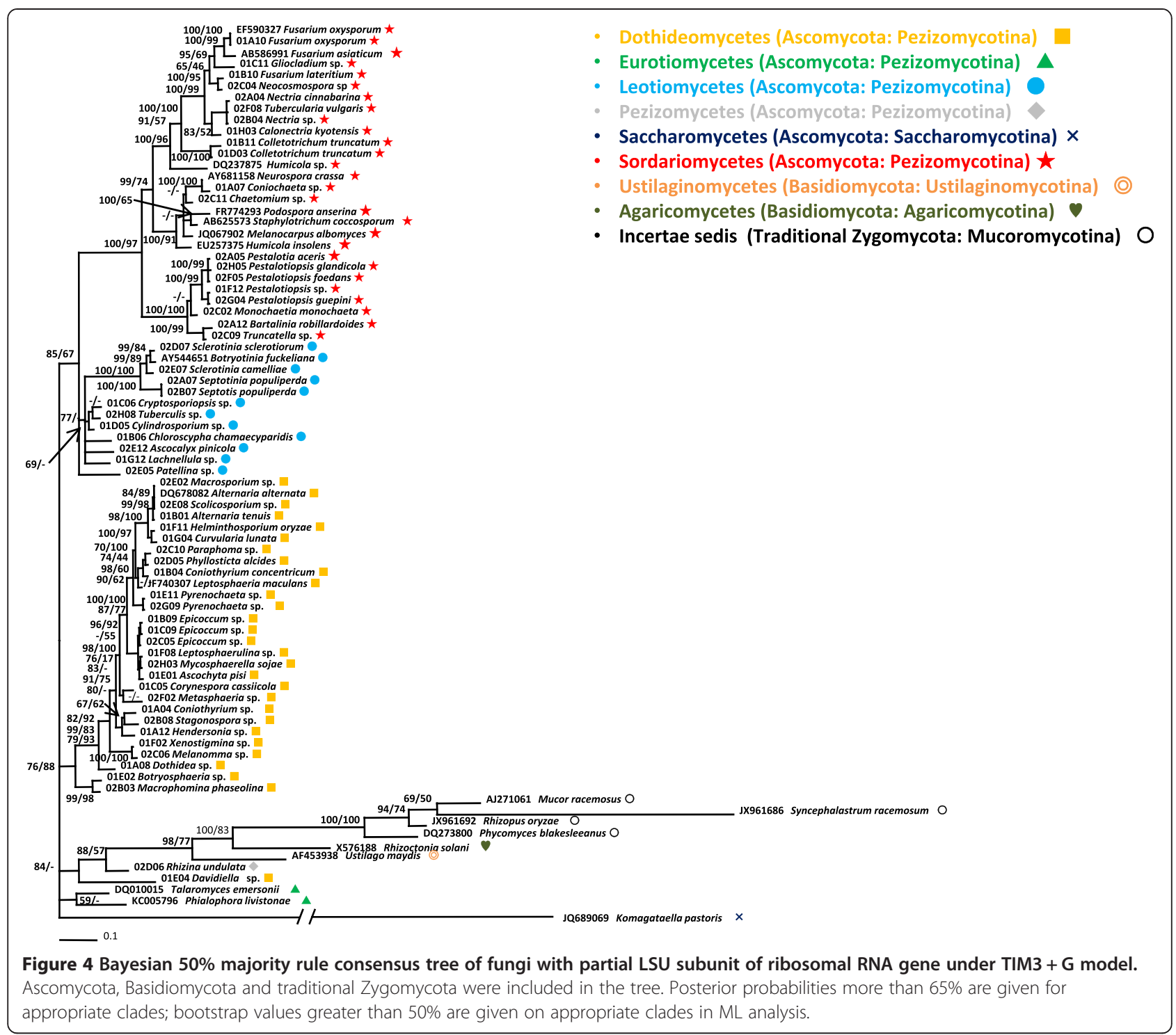

which is most closely related to the nematode sequences and on the basal position of the nematode clade in the tree (labeled as FungiA in Figure 6) consisted of sequences from Sordariomycetes fungi (Figure 5 and Additional file 2: Figure S3). Another fungal clade positioned next to insect clade (labeled as FungiB in Figure 6) was made up of fungi belonging to Mucoromycotina and Basidiomycetes.

\section{Discussion}

In this study we have identified 44 and 70 novel GH45like sequences from nematodes and fungi respectively, as a result of a wide range screening programme. This is equivalent to two thirds of the eukaryotic GH45 genes previously described (CAZy: http://www.cazy.org/). Most of these sequences are from species for which there was no prior knowledge of GH45 cellulases. This study has therefore greatly increased the available information about distribution of $\mathrm{GH} 45$ sequences in eukaryotes.

Our study suggests distribution of GH45 genes in nematodes is likely to be restricted in a single phylogenetic group that includes the families Parasitaphelenchidae and Aphelenchoididae as the sequences have been found only from Bursaphelenchus species, Ruehmaphelenchus sp. and $A$. besseyi. No GH45-like sequence was detected from other nematode species including Pratylenchus sp. and Aphelenchus sp. by PCR amplification in this study and no sequence showing similarity to GH45 genes has been identified in the extensive genome, EST or RNA-seq sequences from any other nematodes including C. elegans, Meloidogyne species and A. avenae. However, there are still many nematode genera, including plant-parasitic species, which have not yet been subjected to detailed analysis. The possibility that other species which 


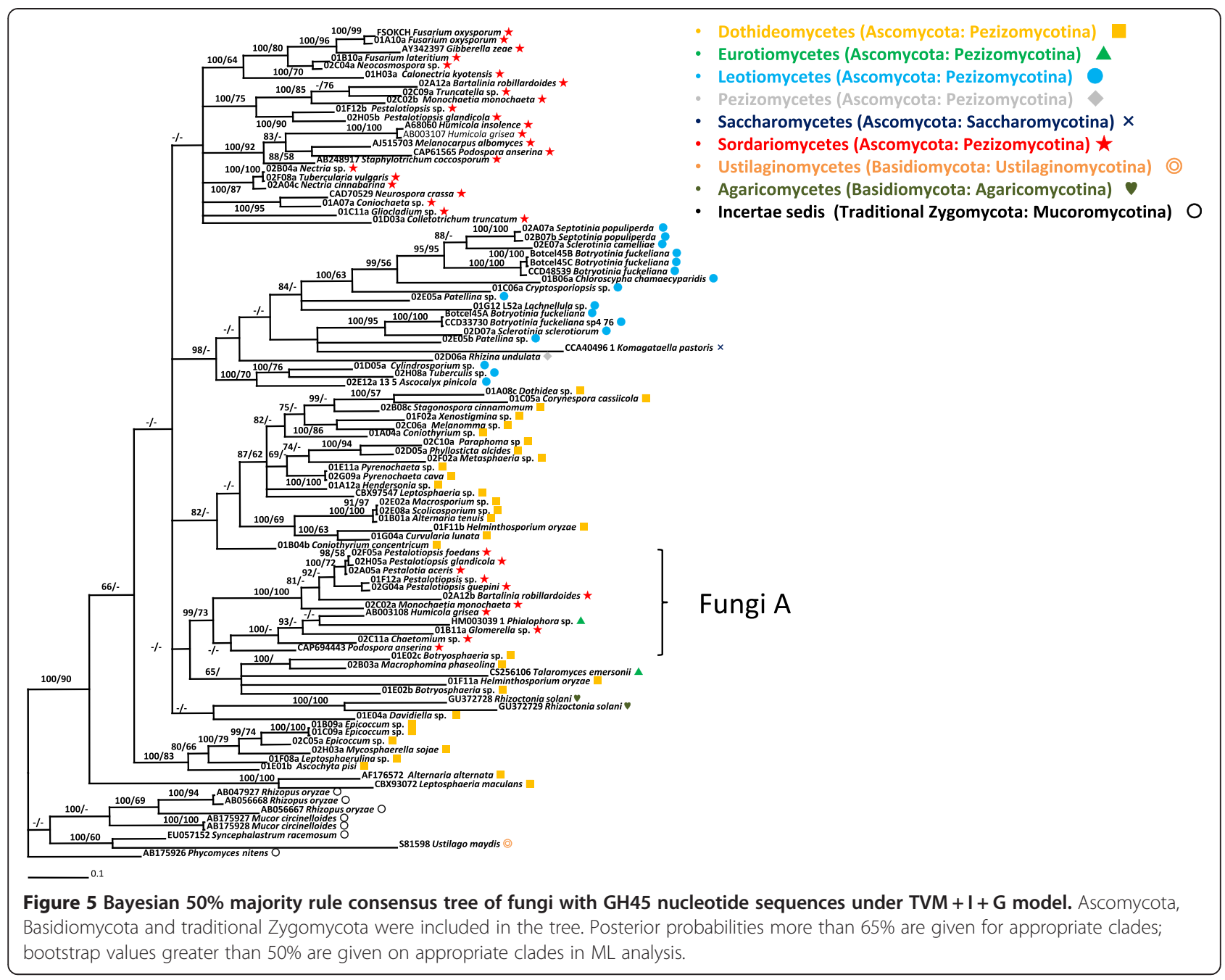

have not yet been analysed also have GH45-like sequences therefore remains.

In fungi, GH45 sequences were found from a variety of Ascomycota species, ranging from Sordariomycetes to Saccharomycetes. Only a small number of GH45 sequences have previously been reported from Basidiomycetous fungi and no PCR amplification of these sequences was observed from any of the Basidiomycetous fungi used in this study, suggesting GH45 genes were more widely distributed in Ascomycota than in Basidiomycota. The phylogenetic trees of fungal GH45 sequences showed good consistency with the LSU tree in terms of species relationships, although some duplications in GH45 sequences were observed in some specific clades and they show a nested structure in the tree. Therefore it seems likely the GH45 genes were inherited vertically and evolved from a common ancestor of these fungi. Currently seven phyla in the kingdom Fungi are proposed: Microsporidia, Chrytridiomycota, Blastocladiomycota, Neocallimastigomycota, Glomeromycota, Ascomycota, and Basidiomycota [19]. In this study we used large numbers of species mainly from two big fungal groups (Ascomycota and Basidiomycota). It would be interesting to investigate distributions of GH45 genes in other phyla as they still remain unclear.

The phylogenetic trees of nematode GH45 sequences showed a more complex structure than those of the fungi. The fact that several different copies of the genes are present within each individual nematode makes it difficult to interpret the phylogenetic trees. For example, we found 4 distinct GH45 sequences in B. luxuriosae and B. purvispicularis and in each case subsets of these sequences are nested with sequences from other species within the trees (Figure 3). In addition we observe several small clusters comprised by multiple sequences from one species within the tree (Figure 3). These patterns are consistent with an initial expansion of the gene family in the common ancestor of the Bursaphelenchus genus followed by further expansions within individual clades and species.

The genome sequence of B. xylophilus revealed the species has 11 GH45 genes in the genome [14] and in our tree they were separated into 5 clusters (Figure 3). This adds further weight to the suggestion that expansions have 


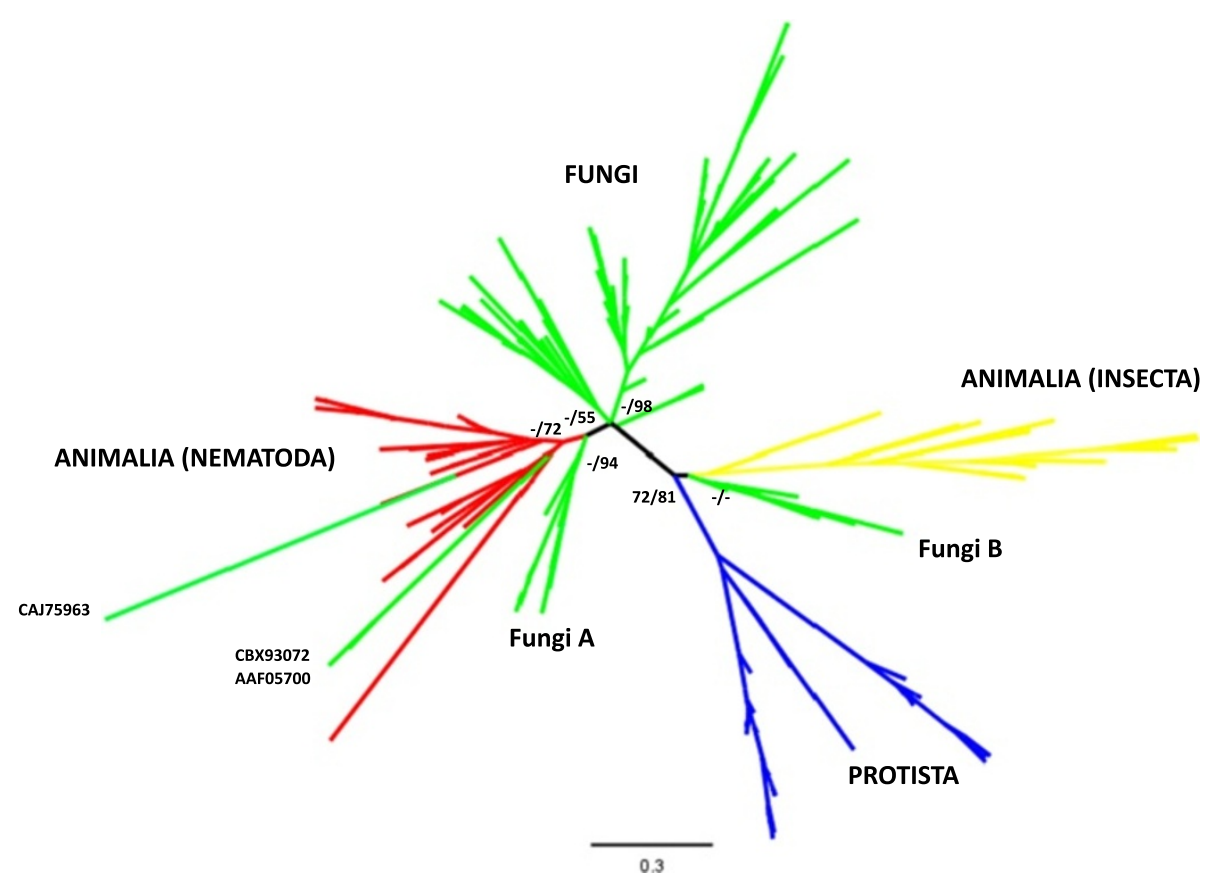

Figure 6 Best tree of Maximum Likelihood analysis using the program RAxML-VI-HPC v. 4.0.0. with GH45 sequences. The amino acid sequences from Animalia, Fungi and Protista Kingdoms were used, and LG + G model was conducted in the analysis. Bootstrap values and posterior probabilities are showed on supported major clades.

occurred both before and after speciation. Some of those genes have another intron position (p8) that was not found in other nematode species using PCR screening, suggesting $\mathrm{p} 8$ genes in other species might be missed in the PCR process possibly because of primer mismatches. A more complete set of cellulase genes of these nematode species will enable a more detailed analysis of the evolution of these genes in nematodes.

Despite the complex structures of the phylogenetic trees generated there is some correlation between the phylogeny based on rDNA SSU sequences and the GH45 trees. A phylogenetic clade in the GH45 trees which includes the xylophilus group and a clade with $B$. kiyoharai and Bursaphelenchus sp2 are both well supported and these clades are also well supported in the tree obtained using SSU rDNA. This again suggests the distribution of GH45 cellulases in Bursaphelenchus species is originated from a common ancestor followed by expansions of the genes during the evolution.

We found two interesting inconsistencies between the GH45 tree and the SSU tree. GH45 sequences of B. parvispicularis are clustered together with those from the xylophilus group while the SSU of the species is phylogenetically closer to bark beetle/weevil associates ( $B$. yongensis, B. poligraphi, Bursaphelenchus sp2 and sp3.). Although we do not have a clear explanation for this inconsistency, this might be related with their biological characters, e.g., carrier insect and habitat environments.
The species in the xylophilus group are associated with cerambycid beetles and inhabit relatively deep wood (in humid conditions), and the other smaller insect associates inhabit shallow wood (in dry conditions). Although the detailed life history of $B$. parvispicularis has not been examined, the habitat preference might be closer to that of the xylophilus group. The detailed biological analysis of $B$. parvispicularis may give an insight into the function of these genes in relation to nematodes' biological characters.

The GH45 sequence from $A$. besseyi is in a closer position to the sequences from the Bursaphelenchus species than those from Ruehmaphelenchus sp. in the tree. As seen in the SSU tree, $A$. besseyi is thought to be more distantly related to Bursaphelenchus species than other Aphelenchus and Ruehmaphelenchus species (Figure 2). Aphelenchoides species are mainly fungal-feeders and are thought to have less association with plants than Bursaphelenchus. However, A. besseyi is one of a few species which are known to be parasitic to plants. It would be interesting to examine GH45 genes in other Aphelenchoides species to have insights into the evolution of parasitism in this nematode genus.

GH45 sequences are widely represented in other organisms including bacteria, protists, insects and molluscs [20]. Sequences from molluscs and bacteria were excluded from this analysis because of their low sequence similarities to GH45 sequences from other species. The 
genes from these groups are thought to comprise a subfamily within the GH45 family. Our broad phylogeny of GH45 sequences showed a clear grouping of organisms in the best tree obtained (Figure 6). Sequences from insects and protists presented monophyletic clades in the tree. Taking into account of the position of nematodes in the tree and the presence of the genes in Ruehmaphelenchus sp. and $A$. besseyi it is possible that the HGT event that gave rise to GH45 sequences in nematodes occurred in an early ancestral species in Aphelenchoidea from a close contact with Ascomycota fungi species as previously suggested $[9,14]$. We hypothesise that the nematodes acquired GH45 genes from one of Sordariomycetous fungi as sequences from these fungi are located at the basal position of the all nematode sequences in the tree (Figure 6). The majority of the Aphelenchoidea species are fungal-feeders and some of the species live in a densely fungal populated environment.

The GH45 sequences from fungal species (CAJ75963 [Rasamsonia emersonii]; CBX93072 [Leptosphaeria maculans]; AAF05700 [Alternaria alternata]) are clustered with the sequences from the Nematoda. This is difficult to explain and may have arisen due to an artifact or, more speculatively, due to sequence changes reflecting functional restrictions on the cellulases within these species.

The nematode GH45 sequences described here have three types of intron positions (p0, p8 and p11). Intriguingly position 11 is shared by the nematodes and some fungi. Although introns have high mutation rates, making it difficult to trace lineages through sequence similarity, their positions are well conserved and can provide strong evidence to support conclusions on the origins of the genes [21]. Therefore the fact that nematode and fungal genes share an intron position in some cases suggests a common origin of these genes. Indeed most of the fungal species (ten out of 15) that possess this intron position belong to Letiomycetes and cluster into one clade in the tree (Figure 4). In addition, the aforementioned sequences from Sordariomycetes fungi (labeled FungiA in Figures 5 and 6 and Additional file 2: Figure S3) also have a position 11 intron as well as genes with no intron. This can be a support for the hypothesis that the source of the nematode genes is likely to be a Sordariomycetes fungus.

Another family of cellulases is represented in other groups of plant-parasitic nematodes. All members of clade 12 of phylum Nematoda analysed to date harbor one or multiple GH5 cellulases [22]. In the Tylenchid plant parasites this family of GH5 cellulases is thought to have been derived from bacteria [22] while in the case of Pristonchus spp. (Clade 9, Diplogasteridae) the GH5 sequences present are more likely to have been acquired from an amoebozoan or related microorganism [23]. The presence of GH5 has also been identified in the fungivorous nematode A. avenae [24]. The phylogenetic position of this species was controversial until recently but now it is clearly accepted that Aphelenchus is more closely related to Tylenchida than to Aphelenchoides and Bursaphelenchus based on the comprehensive molecular phylogenetic study by Van Megen et al. [8]. Our tree also supports this phylogenetic position (Figure 2).

Bursaphelenchus species are likely to have GH45 cellulases regardless of their pathogenicity to plants. The only species proven to be pathogenic in natural conditions from our dataset of studied sequences is B. xylophilus [14], with only a few other species demonstrated as weak disease agents under certain environmental conditions [25]. Most other species, including Ruehmaphelenchus, are associated only with dead trees. Our finding of the widespread occurrence of GH45 cellulases across the Bursaphelenchus genus and in a closely related genus (Ruehmaphelenchus) suggests GH45 cellulases are used by nematodes to soften the cell walls of plants regardless of whether the nematode is a pathogen or simply a fungal feeder that moves through dead plants to locate food.

\section{Conclusions}

It used to be believed that animals (Metazoa) do not have endogenous cellulase (endo-beta-1,4-glucanase) and rely on their symbiotic microorganisms for cellulose digestion. However, it is now clear that some invertebrate species, including nematodes, have endogenous cellulase genes which produce enzymes to digest cellulose.

In order to investigate distribution and evolution of GH45 cellulase genes in nematodes and fungi we performed a wide ranging screen and intensive phylogenetic analysis of GH45 sequences. We identified 44 novel sequences from a small group of nematode species and 77 from a wide variety of Ascomycetous fungi, indicating a wide distribution of GH45 cellulases in Ascomycetous fungi and so far been found in a single major nematode lineage. The close relationships between the sequences from nematodes and Ascomycetous fungi, as well as the conserved gene structures gave us the reasonable hypothesis that nematode GH45 cellulase genes were acquired via HGT from fungi probably belonging to class Sordariomycetes.

\section{Methods}

\section{Biological materials}

The fungal strains and nematode species used in this study were from the culture collection stored at the Forest Pathology Lab in FFPRI or from NIAS Genebank culture collection (Additional file 1: Table S1, S2).

\section{DNA extraction}

Fungi were cultured on cellophane membranes placed on potato dextrose agar (PDA, Eiken Chemical) plates at $23^{\circ} \mathrm{C}$ for periods appropriate for each fungus. Fungal 
mycelium was harvested from each plate by scratching the surface of the membranes using small metal spatulas. The mycelium was either immediately used for DNA extraction or stored at $-80^{\circ} \mathrm{C}$ for further use. Genomic DNAs from fungi were extracted using a rapid and highthroughput extraction method [26].

Nematodes were cultured at $25^{\circ} \mathrm{C}$ on Botrytis cinerea grown on potato dextrose agar plates. Nematodes were collected using a modified Baermann funnel technique [27] and cleaned in several rinses of 0.5x PBST before use. Genomic DNAs were extracted as described in Kikuchi et al. [26].

\section{PCR amplification and Sequencing}

Two degenerate primers, GHF45-1f and GHF45-2r were designed from a highly conserved region of GHF45 cellulases selected from CAZy homepage (Carbohydrated Active EnZYmes; http://www.cazy.org/). The sequence of GHF45-1f is based on TRYWDCC (amino acids 22-28 in unprocessed B. xylophilus Bx-ENG1). The sequence of GHF45-2r is based on PGGG(F/V)GA (amino acids 141147 in unprocessed B. xylophilus Bx-ENG1) (Additional file 2: Figure S1).

Amplification was performed using GoTaq green master mix (Promega) or IQ SYBR Green Supermix (BioRad) with $0.5 \mu \mathrm{M}$ of each primer and appropriately diluted genomic DNA solution. After checking the bands on a $1 \%$ agarose gel, PCR products were cloned into pGEM-Teasy vector (Promega) and transformed into E. coli (DH5alpha). Sixteen $E$. coli clones from each product were picked randomly from the plate and sequenced from both ends using BigDye Terminator ver 3.1 (Life Technologies).

The small subunit of ribosomal RNA genes of nematodes were amplified using primers F07 (5'-AAAGAT TAAGCCATGCATG-3') and nR (5' ${ }^{\prime}$-TTACGACTTTT GCCCGGTTC-3'). Large subunit of ribosomal RNA genes of fungi were amplified with primer LROR and LR5 (a location map and oligonucleotide sequences of these primers can be found at http://www.biology.duke. edu/fungi/mycolab/primers.htm). Amplified products were cleaned with Minelute 96 plate (Qiagen) and sequenced from the both ends.

\section{Intron prediction and RT-PCR}

Intron prediction was performed using SpliceView (http://www.itb.cnr.it/webgene/) with an option of organism="Caenorhabditis elegans" or organism="Aspergillus niger" for nematodes and fungi respectively and manually adjusted on the basis of conserved regions of GH45 cellulase sequences.

RNA was extracted from nematodes which were cultured on B. cinerea as described in Kikuchi et al. [28] and cDNA was synthesized using iScript (BioRad) following the manufacturer's instructions. The GH45 cellulase cDNA was PCR amplified using primers designed for the genomic DNA fragment and sequenced to confirm the exon/intron structures of the fragments.

\section{Phylogenetic analyses}

GH45 cellulase sequences were obtained from the CAZy homepage (http://www.cazy.org) and GenBank database in addition to the sequences obtained in this study. Specific phylogenies of nucleotides and amino acids from GH45 coding sequences were studied in the Bursaphelenchus genus using Ruehmaphelenchus sp. as an outgroup, while for the fungi phylogeny Phycomyces nitens was used as an outgroup. A combined phylogenic tree was also constructed using 208 GH45 amino acid sequences (including all sequences obtained in this study). Bacteria and mollusc sequences were excluded from the analysis. Phylogenetic trees based on ribosomal sequences from fungi and nematodes were made using sequences obtained in this study and sequences from GenBank. Nucleotide and amino acid sequences for the different phylogenetic analysis were aligned using ClustalX2 [29] and MUSCLE [30], respectively. The best fitting model of protein and DNA evolution were obtained based on the AIC (Akaike Information Criterion) using ProtTest 2.4 server [31] and jModelTest v. 2 [32], respectively. Models for the different alignments were as follows: (i) Nematode ribosomal sequences: GTR + I + G; (ii) Nematode GH45 DNA sequences: 010234 + I + G + F; (iii) Nematode GH45 aminoacid sequences: WAG + I + G + F; (iv) Fungi ribosomal sequences: TIM3 + G; (v) Fungi GH45 DNA sequences: TVM + I + G; (vi) Fungi GH45 aminoacid sequences: LG + I+ G. Phylogenetic analysis of the sequence data sets were performed with maximum likelihood (ML) using the program RAxML-VI-HPC v. 4.0.0 (Randomized Accelerated Maximum Likelihood for High Performance Computing) [33] using 500 bootstraps and the computed model. Bayesian inference (BI) was conducting using MrBayes 3.1.2 [34] using aforementioned site-specific models. Four chains were run for a minimum of $4 \times 10^{6}$ generations and two independent runs were performed. After discarding burnin samples and evaluating convergence, the remaining topologies were used to generate a $50 \%$ majority-rule consensus tree. Trees were visualised using FigTree v1.4.0 (http://tree.bio.ed.ac.uk/software/figtree/).

\section{Availability of supporting data}

Sequences obtained in this study have been deposited to Genbank under accession nos: KF590043-KF590214.

\section{Additional files}

Additional file 1: Table S1. Nematode cultures used in this study. Table S2. Fungi cultures used in this study. Table S3. Nematode GH45 sequences obtained in this study. Table S4. Fungal GH45 sequences 
obtained in this study. Table S5. Sequences and Genbank accession numbers used in Figure 4. Table S6. Species names and GenBank accession numbers used in Figure 6.

Additional file 2: Figure S1. Amino acid alignment of $\mathrm{GH} 45$ proteins and intron positions. Intron positions are indicated by triangles on the alignment. Conserved regions used to design primers are boxed. Asterisks indicate the two catalytic core residues (Asp, Asp). The numbers to the left indicate the amino acid position of the respective proteins. Phase of the introns is shown by distinct triangles. BXENG1, 2 and $3-B$. xylophilus sequences (nematode - BAD34543-5), B_ciner1 - GHF45 cellulase from Botrytis cinerea (fungus - CCD33730), R.oryza1 - Rhizopus oryzae (fungus - BAC53956), H_insol1 - Humicola insolens (fungus CAB42307), A.germ1 - Apriona germari (insect - AAN78326). Figure S2. Bayesian 50\% majority rule consensus tree of $\mathrm{GH} 45$ amino acid sequences from Aphelenchoidea under WAG + I $G+F$ model. Posterior probabilities more than $65 \%$ are given for appropriate clades; bootstrap values greater than $50 \%$ are given on appropriate clades in ML analysis. Figure S3. Bayesian 50\% majority rule consensus tree of $\mathrm{GH} 45$ amino acid sequences from Ascomycota, Basidiomycota and Zygomycota under $\mathrm{LG}+\mathrm{I}+\mathrm{G}$ model. Posterior probabilities more than $65 \%$ are given for appropriate clades; bootstrap values greater than $50 \%$ are given on appropriate clades in $\mathrm{ML}$ analysis.

\section{Abbreviations}

Bursaphelenchus sp. 3 is now described as Bursaphelenchus niphades [15]; GH: Glycoside hydrolase; HGT: Horizontal gene transfer.

\section{Competing interests}

The authors declare that they have no competing interests.

\section{Authors' contributions}

JEP and $\mathrm{YH}$ carried out the phylogenetic analyses, participated in drafting the manuscript. JIT and AH carried out gene structure analysis and drafted the manuscript. HM and $\mathrm{NH}$ participated in the phylogenetic analyses. JTJ and TK conceived of the study, participated in its design and coordination and drafted the manuscript. All authors read and approved the final manuscript.

\section{Acknowledgements}

The authors thank Asuka Shichiri for her valuable technical assistance and Yuko Ota for providing fungal cultures. This work was supported by JSPS KAKENHI Grant Numbers 20353659 and 23248024. JEPR and IJT were supported by JSPS Postdoctoral Fellowship Program for Foreign Researchers. The James Hutton Institute receives funding from the Scottish Government. Part of this work was funded by ERASMUS MUNDUS programme 2008-102 (EUMAINE).

\section{Author details}

${ }^{1}$ Division of Parasitology, Faculty of Medicine, University of Miyazaki, Miyazaki 889-1692, Japan. ${ }^{2}$ Instituto de Agricultura Sostenible (IAS), Consejo Superior de Investigaciones Científicas (CSIC), Campus de Excelencia Internacional, Apdo. 4084, 14080 Córdoba, Spain. ${ }^{3}$ Forestry and Forest Products Research Institute, Tsukuba, Ibaraki 305-8687, Japan. ${ }^{4}$ Biodiversity (Mycology), Eastern Cereal and Oilseed Research Centre, Agriculture and Agri-Food Canada, Ottawa, ON K1A0C6, Canada. ${ }^{5}$ James Hutton Institute, Invergowrie, Dundee DD2 5DA, UK. ${ }^{6}$ Biology Department, Ghent University, K.L. Ledeganckstraat 35, 9000 Ghent, Belgium.

Received: 20 November 2013 Accepted: 17 March 2014 Published: 1 April 2014

\section{References}

1. Smant G, Stokkermans JP, Yan Y, de Boer JM, Baum TJ, Wang X, Hussey RS, Gommers FJ, Henrissat B, Davis EL, Helder J, Schots A, Bakker J: Endogenous cellulases in animals: isolation of beta-1, 4-endoglucanase genes from two species of plant-parasitic cyst nematodes. Proc Natl Acad Sci U S A 1998, 95(9):4906-4911.

2. Watanabe H, Noda H, Tokuda G, Lo N: A cellulase gene of termite origin. Nature 1998, 394(6691):330-331.
3. Henrissat B, Bairoch A: New families in the classification of glycosyl hydrolases based on amino acid sequence similarities. Biochem J 1993, 293(Pt 3):781-788.

4. Henrissat B, Callebaut I, Fabrega S, Lehn P, Mornon JP, Davies G: Conserved catalytic machinery and the prediction of a common fold for several families of glycosyl hydrolases. Proc Natl Acad Sci 1995, 92(15):7090-7094.

5. Yan Y, Smant G, Stokkermans J, Qin L, Helder J, Baum T, Schots A, Davis E: Genomic organization of four beta-1,4-endoglucanase genes in plant-parasitic cyst nematodes and its evolutionary implications. Gene 1998, 220(1-2):61-70.

6. Jones JT, Furlanetto C, Kikuchi T: Horizontal gene transfer from bacteria and fungi as a driving force in the evolution of plant parasitism in nematodes. Nematology 2005, 7(5):641-646.

7. Haegeman A, Jones JT, Danchin EG: Horizontal gene transfer in nematodes: a catalyst for plant parasitism? Mol Plant Microbe Interact 2011, 24(8):879-887.

8. van Megen $H$, van den Elsen S, Holterman M, Karssen G, Mooyman P, Bongers T, Holovachov O, Bakker J, Helder J: A phylogenetic tree of nematodes based on about 1200 full-length small subunit ribosomal DNA sequences. Nematology 2009, 11(6):927-950.

9. Kikuchi T, Jones JT, Aikawa T, Kosaka H, Ogura N: A family of glycosyl hydrolase family 45 cellulases from the pine wood nematode Bursaphelenchus xylophilus. FEBS Lett 2004, 572(1-3):201-205.

10. Shibuya $H$, Kikuchi T: Purification and characterization of recombinant endoglucanases from the pine wood nematode Bursaphelenchus xylophilus. Biosci Biotechnol Biochem 2008, 72(5):1325-1332.

11. Gao B, Allen R, Davis EL, Baum TJ, Hussey RS: Molecular characterisation and developmental expression of a cellulose-binding protein gene in the soybean cyst nematode Heterodera glycines. Int J Parasitol 2004, 34(12):1377-1383.

12. Jones JT, Moens M, Mota M, Li H, Kikuchi T: Bursaphelenchus xylophilus: opportunities in comparative genomics and molecular host-parasite interactions. Mol Plant Pathol 2008, 9(3):357-368.

13. Kikuchi T, Cock PJA, Helder J, Jones JT: Characterisation of the transcriptome of Aphelenchoides besseyi and identification of a GHF 45 cellulase. Nematology 2014, 16(1):99-107.

14. Kikuchi T, Cotton JA, Dalzell JJ, Hasegawa K, Kanzaki N, McVeigh P, Takanashi T, Tsai IJ, Assefa SA, Cock PJA, Otto TD, Hunt M, Reid AJ, Sanchez-Flores A, Tsuchihara K, Yokoi T, Larsson MC, Miwa J, Maule AG, Sahashi N, Jones JT, Berriman M: Genomic Insights into the Origin of Parasitism in the Emerging Plant Pathogen Bursaphelenchus xylophilus. PLoS Pathog 2011, 7(9):e1002219.15.

15. Tanaka ES, Tanaka R, Akiba M, Aikawa T, Maehara N, Takeuchi Y, Kanzaki N: Bursaphelenchus niphades n. sp. (Tylenchina: Aphelenchoididae) amensally associated with Niphades variegatus (Roelofs) (Coleoptera: Curculionidae). Nematology 2014, 16(3):259-281.

16. Spatafora JW, Sung GH, Johnson D, Hesse C, O'Rourke B, Serdani M, Spotts R, Lutzoni F, Hofstetter V, Miadlikowska J, Reeb V, Gueidan C, Fraker E, Lumbsch T, Lücking R, Schmitt I, Hosaka K, Aptroot A, Roux C, Miller AN, Geiser DM, Hafellner J, Hestmark G, Arnold AE, Büdel B, Rauhut A, Hewitt D, Untereiner WA, Cole MS, Scheidegger $C$, et al: A five-gene phylogeny of Pezizomycotina. Mycologia 2006, 98(6):1018-1028.

17. James TY, Kauff F, Schoch CL, Matheny PB, Hofstetter V, Cox CJ, Celio G, Gueidan C, Fraker E, Miadlikowska J, Lumbsch HT, Rauhut A, Reeb V, Arnold AE, Amtoft A, Stajich JE, Hosaka K, Sung GH, Johnson D, O'Rourke B, Crockett M, Binder M, Curtis JM, Slot JC, Wang Z, Wilson AW, Schüssler A, Longcore JE, O'Donnell K, Mozley-Standridge S: Reconstructing the early evolution of Fungi using a six-gene phylogeny. Nature 2006, 443(7113):818-822.

18. Schoch CL, Sung GH, Lopez-Giraldez F, Townsend JP, Miadlikowska J, Hofstetter V, Robbertse B, Matheny PB, Kauff F, Wang Z, Gueidan C, Andrie RM, Trippe K, Ciufetti LM, Wynns A, Fraker E, Hodkinson BP, Bonito G, Groenewald JZ, Arzanlou M, de Hoog GS, Crous PW, Hewitt D, Pfister DH, Peterson K, Gryzenhout M, Wingfield MJ, Aptroot A, Suh SO, Blackwell M: The Ascomycota tree of life: a phylum-wide phylogeny clarifies the origin and evolution of fundamental reproductive and ecological traits. Syst Biol 2009, 58(2):224-239.

19. Hibbett DS, Binder M, Bischoff JF, Blackwell M, Cannon PF, Eriksson OE, Huhndorf S, James T, Kirk PM, Lucking R, Thorsten Lumbsch H, Lutzoni F, Matheny PB, McLaughlin DJ, Powell MJ, Redhead S, Schoch CL, Spatafora JW, Stalpers JA, Vilgalys R, Aime MC, Aptroot A, Bauer R, Begerow D, Benny GL, 
Castlebury LA, Crous PW, Dai YC, Gams W, et al. A higher-level phylogenetic classification of the Fungi. Mycol Res 2007, 111(Pt 5):509-547.

20. Todaka N, Inoue T, Saita K, Ohkuma M, Nalepa CA, Lenz M, Kudo T, Moriya S: Phylogenetic Analysis of Cellulolytic Enzyme Genes from Representative Lineages of Termites and a Related Cockroach. PLOS ONE 2010, 5(1):e8636.

21. Krauss V, Thummler C, Georgi F, Lehmann J, Stadler PF, Eisenhardt C: Near intron positions are reliable phylogenetic markers: an application to holometabolous insects. Mol Biol Evol 2008, 25(5):821-830.

22. Rybarczyk-Mydlowska K, Maboreke HR, van Megen $\mathrm{H}$, van den Elsen $\mathrm{S}$, Mooyman P, Smant G, Bakker J, Helder J: Rather than by direct acquisition via lateral gene transfer, GHF5 cellulases were passed on from early Pratylenchidae to root-knot and cyst nematodes. BMC Evol Biol 2012 12(1):221.

23. Mayer W, Schuster L, Bartelmes G, Dieterich C, Sommer R: Horizontal gene transfer of microbial cellulases into nematode genomes is associated with functional assimilation and gene turnover. BMC Evol Biol 2011, 11(1):13.

24. Karim N, Jones JT, Okada H, Kikuchi T: Analysis of expressed sequence tags and identification of genes encoding cell-wall-degrading enzymes from the fungivorous nematode Aphelenchus avenae. BMC Genomics 2009, 10(1):525

25. Polomski J, Rigling D: Effect of Watering Regime on Disease Development in Pinus sylvestris Seedlings Inoculated with Bursaphelenchus vallesianus and B. mucronatus. Plant Dis 2010, 94(8):1055-1061.

26. Kikuchi T, Karim N, Masuya H, Ota Y, Kubono T: An inexpensive high-throughput method to extract high yields of good quality DNA from fungi. Mol Ecol Resour 2009, 9:41-45.

27. Southey JF: Laboratory methods for work with plant and soil nematodes. 6th edition. London: HMSO Books; 1986

28. Kikuchi T, Aikawa T, Kosaka H, Pritchard L, Ogura N, Jones JT: Expressed sequence tag (EST) analysis of the pine wood nematode Bursaphelenchus xylophilus and B. mucronatus. Mol Biochem Parasitol 2007, 155(1):9-17.

29. Larkin MA, Blackshields G, Brown NP, Chenna R, McGettigan PA McWilliam H, Valentin F, Wallace IM, Wilm A, Lopez R, Thompson JD, Gibson TJ, Higgins DG: Clustal W and Clustal X version 2.0. Bioinformatics 2007, 23(21):2947-2948.

30. Edgar RC: MUSCLE: multiple sequence alignment with high accuracy and high throughput. Nucleic Acids Res 2004, 32(5):1792-1797.

31. Abascal F, Zardoya R, Posada D: ProtTest: selection of best-fit models of protein evolution. Bioinformatics 2005, 21(9):2104-2105.

32. Darriba D, Taboada GL, Doallo R, Posada D: jModelTest 2: more models, new heuristics and parallel computing. Nat Methods 2012, 9(8):772

33. Stamatakis A, Hoover P, Rougemont J: A rapid bootstrap algorithm for the RAxML Web servers. Syst Biol 2008, 57(5):758-771.

34. Huelsenbeck JP, Ronquist F: MRBAYES: Bayesian inference of phylogenetic trees. Bioinformatics 2001, 17(8):754-755.

doi:10.1186/1471-2148-14-69

Cite this article as: Palomares-Rius et al: Distribution and evolution of glycoside hydrolase family 45 cellulases in nematodes and fungi. BMC Evolutionary Biology 2014 14:69

\section{Submit your next manuscript to BioMed Central and take full advantage of:}

- Convenient online submission

- Thorough peer review

- No space constraints or color figure charges

- Immediate publication on acceptance

- Inclusion in PubMed, CAS, Scopus and Google Scholar

- Research which is freely available for redistribution 